\title{
Numerical Investigations of Laminar Buoyant Heat Transfer in a 2D-Enclosure - Application to Wind Turbine Nacelle Operating in Hot Climate
}

\author{
M. A. MAHDI*,**, A. SMAILI* \\ *Laboratoire de Génie Mécanique et Développement, Ecole Nationale Polytechnique, P.B. 182 \\ El-Harrach, 16200, Algeria, E-mail: arezki.smaili@g.enp.edu.dz \\ **University of Minnesota, Minneapolis, Minnesota, 55455, USA, E-mail: mahdi012@umn.edu \\ cross $^{\text {ref }}$ http://dx.doi.org/10.5755/j01.mech.23.5.15815
}

\section{Introduction}

The climate of the Algerian Sahara is characterized by extreme conditions. Wind turbines that would be installed in this region, namely the electromechanical equipment located inside the nacelle (e.g. electrical generator) are subjected to high and fluctuating temperature gradients during the day and seasons. This reality might generate contradictory constraints of design and can lead to some difficulties of conception. In order to maintain an appropriate temperature of the air inside the nacelle, a cooling system must be employed, so the heat released by the generator could be rejected towards the atmosphere. The resulting heat transfer must be controlled properly to ensure safe operation and to prevent failure of the wind turbine, under high ambient temperature conditions. Thus, a compromise should be found between the air temperature levels inside the nacelle on one hand, and the high cooling efficiency which leads to lower reliability and higher cost for such a complex cooling system, on the other hand. The main challenge for electronic equipment in a wind turbine nacelle is that they must withstand a wide range of ambient temperature, usually from $-20{ }^{\circ} \mathrm{C} \mathrm{C}$ to $+55^{\circ} \mathrm{C}$. To assess the effect of the environment temperature on wind turbine nacelle operating in Canadian Nordic climate, a numerical method has been developed previously by Smaili et al. [1].

The air flow has been described by Reynolds averaged NavierStokes equations and the energy equation has been used to account for the heat transfer effects. The resulting governing equations have been solved by the ControlVolume Finite Element Method (CVFEM) [2]. In a recent work [3], a numerical method has been proposed to investigate the nacelle thermal behavior operating under extreme Saharan hot weather conditions. A few studies on the nacelle thermal behavior are available in the literature $[4,6]$, while several works on the heat transfer in rectangular/square enclosures have been published (e.g. [7]). The main purpose of the present study is to assess the cooling capacity needed to ensure acceptable temperature levels inside the nacelle, by considering the buoyancy effect within the nacelle. Hypothetical nacelle has been considered and a boundary layer analysis has been carried out in order to validate the adopted numerical method. Detailed results including temperature and velocity fields within the nacelle have been presented and discussed. To assess properly the buoyancy effect, average temperature distributions inside the nacelle, as well as the required cooling capacity have been investigated.

\section{Mathematical model}

\subsection{Physical problem}

In the present study, we consider a hypothetical nacelle having dimensions with scaling factor of 1/40 from the real dimensions. Fig. 1 illustrates the geometry of the 2Dproblem. The nacelle is considered air-tight, therefore the natural convection problem within the nacelle can be treated independently from the external conditions.

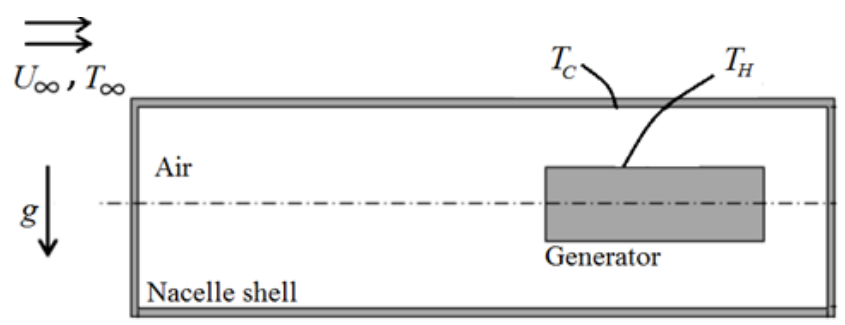

Fig. 1 D-configuration of the physical problem

The following considerations and simplifying assumptions are considered.

- The 2D-case is considered in the present work.

- External heat exchange and air flow around the nacelle are neglected.

- The gravity impact (buoyancy force) is considered. Thus, heat transfer by natural convection takes place within the nacelle.

- Heat transfer by radiation is neglected.

- Heat generation (considered to be resulting mainly from electrical generator) is idealized as an isothermal condition, represented by a hot plate (i.e. generator wall) at temperature $T_{H}$ in the computational domain.

- The cooling system is idealized as an isothermal condition, represented by a cold plate (i.e. nacelle internal wall) at temperature $T_{C}$, which represents the cooling temperature.

- Only laminar natural convection heat transfer regime is considered for this purpose.

\subsection{Governing equations and boundary conditions}

For steady flow conditions, the complete set of fluid equations, expressed in the two dimensional coordinate system, $(x, y)$, consists of the continuity, Navier- 
Stokes and energy equations where Boussinesq approximation is considered for the temperature density dependence.

$$
\begin{aligned}
& \frac{\partial u}{\partial x}+\frac{\partial v}{\partial y}=0, \\
& u \frac{\partial u}{\partial x}+v \frac{\partial u}{\partial y}=-\frac{1}{\rho} \frac{\partial p}{\partial x}+v\left(\frac{\partial^{2} u}{\partial x^{2}}+\frac{\partial^{2} u}{\partial y^{2}}\right), \\
& u \frac{\partial v}{\partial x}+v \frac{\partial v}{\partial y}=-\frac{1}{\rho} \frac{\partial p}{\partial y}+v\left(\frac{\partial^{2} v}{\partial x^{2}}+\frac{\partial^{2} v}{\partial y^{2}}\right)+g \beta\left(T-T_{0}\right), \\
& u \frac{\partial T}{\partial x}+v \frac{\partial T}{\partial y}=\alpha\left(\frac{\partial^{2} T}{\partial x^{2}}+\frac{\partial^{2} T}{\partial y^{2}}\right),
\end{aligned}
$$

where $u$ and $v$ are velocity components in $x$ and $y$ directions. $T$ and $p$ are air temperature and pressure, respectively. $\rho, v$ and $\alpha$ denote respectively density, kinematic viscosity and thermal diffusivity of the air. $g$ is the acceleration due to gravity and $\beta$ the thermal expansion coefficient of the air. $T_{0}$ denotes the reference temperature given by $T_{0}=\left(T_{H}+T_{C}\right) / 2$.

Fig. 2 illustrates the computational domain and the associated boundary conditions, where dimensions are given in millimeter. For the temperature field, the isothermal conditions have been prescribed for nacelle internal wall (i.e. cold plate at $T_{C}$ ) and for the electrical generator wall (i.e. hot plate at $T_{H}$ ). The nonslip conditions are prescribed for velocity field. For simplification reasons, hot plate temperature $T_{H}$ is maintained unchanged at $100^{\circ} \mathrm{C}$ in all this study. The temperature span $\Delta T=T_{H}-T_{C}$ is controlled only by varying cold plate temperature $T_{C}$.

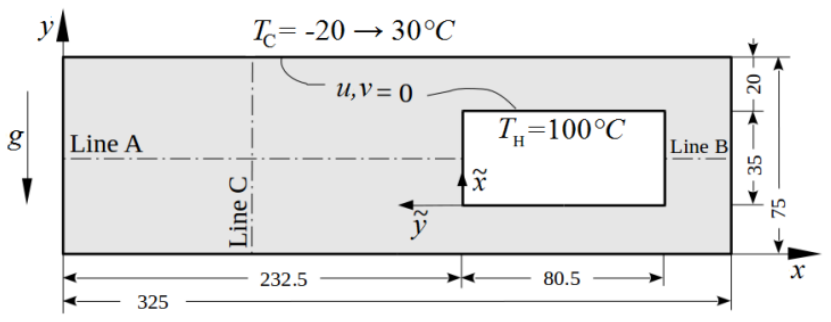

Fig. 2 Computational domain and boundary conditions

It is of interest to notice that for such dimensions scaling of the nacelle and for temperature span $\Delta T$ varying in the range of $120^{\circ} \mathrm{C} \rightarrow 70^{\circ} \mathrm{C}$, Rayleigh number based on the longest distance between hot and cold plates, that is Line A length, is $R a_{A}<\approx 10^{8}$ which, theoretically, would keep the air flow within the nacelle in the laminar regime range.

\section{Numerical method}

\subsection{Numerical scheme}

To solve the resulting governing equations along with the specified boundary conditions, ANSYS FLUENT code based on the finite-volume method has been used. SIMPLE algorithm was selected for the pressure-velocity coupling where the discretized equations have been iterated with relaxation factors of 0.3 for pressure, 0.7 for momentum and 1 for energy as the defaults. The second-order upwind differencing scheme has been adopted for momentum and energy equations.

\subsection{Grid topology and mesh dependence study}

Non-uniform triangular meshes have been used to carry out simulations. Fine grid is considered close to the isothermal walls where high temperature and velocity gradients take place. Fig. 3 illustrates the topology of the grid.

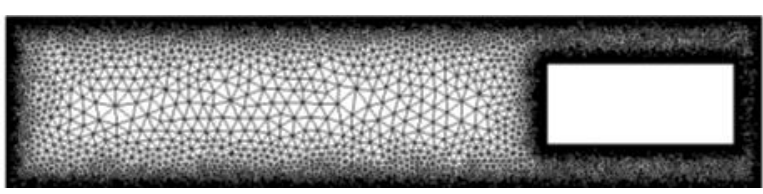

Fig. 3 Grid topology

To determine the appropriate grid size that would produce mesh-independent numerical results, simulations have been performed for typical cold plate and hot plate temperatures. The resulting total heat rate, $q_{\text {in }}$, evaluated by the defining equation (5), has been calculated by considering different grid dimensions, where grids are characterized by the number of nodes that discretizes the computational domain.

$$
q_{\text {in }}=\int_{\Gamma} k\left(\frac{\partial T}{\partial n}\right)_{\text {hot_wall }} d s,
$$

where: $k$ is the air thermal conductivity assumed to be constant and calculated at the mean temperature $\left(T_{H}+T_{C}\right) / 2$. $\Gamma$ denotes the curvilinear path describing the generator wall. $n$ and $s$ are, respectively, the local coordinate normal to the wall and the curvilinear coordinate. Fig. 4 shows the mesh dependence study results. As it can be seen, for number of nodes greater than $6.10^{4}$, the resulting total heat rate seems to be independent of grid size.

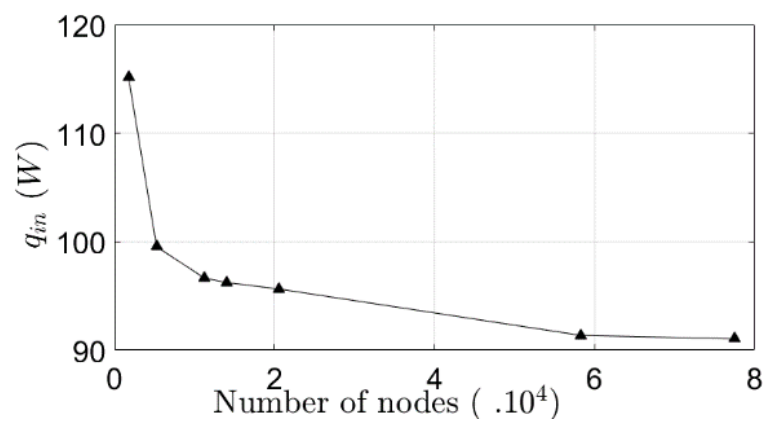

Fig. 4 Mesh dependence study

\section{Results and discussions}

\subsection{Temperature field and streamline contours}

Figs. 5 and 6 show the resulting temperature field and streamline contours obtained for cold and hot plate temperatures of $T_{C}=0^{\circ} \mathrm{C}$ and $T_{H}=100^{\circ} \mathrm{C}$, respectively. As it 
can be seen, the behavior of these simulation results seems to be quite reasonable, thus confirming, qualitatively, the validity of the proposed numerical method, in particular, the concentration of the hot air in the upper part of the nacelle which is due to the decrease in its density, thus confirming the suitability of the adopted Boussinesq approximation.

From Fig. 6, it can be seen that the air motion within the nacelle is characterized by recirculating flow concentrated mainly in three cells adjoining the hot and cold walls. On the left-bottom side of the nacelle, thermal instability starts to occur, giving birth to secondary air circulation core.

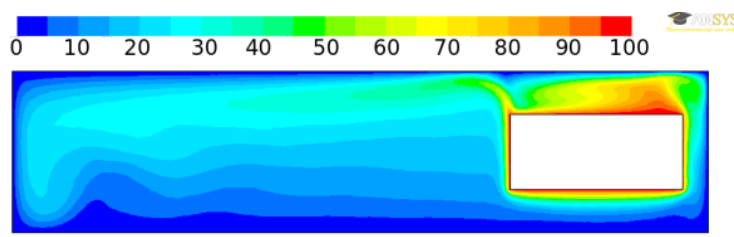

Fig. 5 Temperature distribution within the nacelle $\left({ }^{\circ} \mathrm{C}\right)$

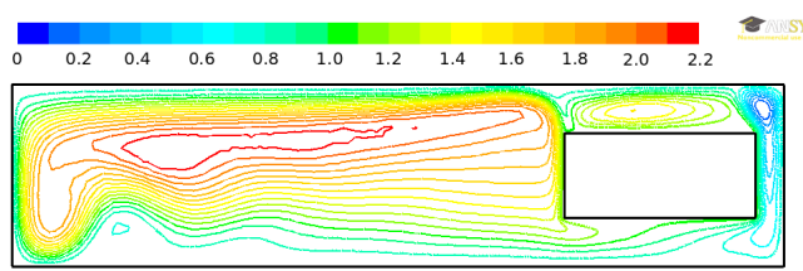

Fig. 6 Streamline contours within the nacelle $\left(.10^{-3} \mathrm{~kg} / \mathrm{s}\right)$

Similar conclusions can be drawn from the results (not presented here) obtained for other temperature spans between hot and cold plates.

\subsection{Temperature and velocity profiles}

To visualize explicitly temperature and velocity fields within the nacelle, results have been extracted along two longitudinal lines, A and B, and one transversal line, $\mathrm{C}$, (cf. Fig. 2) by considering the cold plate temperature varying from $T_{C}=-20^{\circ} \mathrm{C}$ to $30^{\circ} \mathrm{C}$. The obtained profiles of temperature, vertical and horizontal velocity are presented in Fig. 7.

Figs. 7, a and b show profiles of temperature and vertical velocity along Line A, respectively. For different cold plate temperatures, temperature profiles behave similarly, decreasing from the common hot plate temperature $T_{H}$ to the corresponding cooling temperature $T_{C}$. High air motion is noticed, upward in the vicinity of the hot plate and downward near the cold plate. However, air is less active near the cold plate than near the hot plate; $\left|\overline{v_{\max }^{C}}\right| \approx 0.89\left|\overline{v_{\text {max }}^{H}}\right|$ , where the subscripts $H$ and $C$ refer to hot and cold plate vicinities respectively, while the upper-bar denotes the average over the cold plate temperatures range. It is important to notice that boundary layer effect occurs more clearly in the vicinity of the hot plate than on the opposite side where instability of the flow starts to occur.

Along Line B, temperature and vertical velocity profiles behave similarly to those along Line A (cf. Fig. 7, c and d). Nevertheless, the flow is more stable, due to the nearness of hot and cold plates along Line $\mathrm{B}$. The relative shift of maximum vertical velocity between hot and cold plate vicinities is more remarkable along Line B; $\left|\overline{v_{\max }^{C}}\right| \approx 0.51\left|\overline{v_{\max }^{H}}\right|$. Furthermore, it is of interest to notice that, along Line B, for different cold plate temperatures, the corresponding absolute difference between maximum velocities near the hot plate is twice more important than near thecold plate; $\Delta\left|v_{\max }^{C}\right| \approx 0.5 \Delta\left|v_{\max }^{H}\right|$, which means that air motion in the vicinity of hot plate is more sensible to the temperature gap between hot and cold plates, i.e., $\Delta T=T_{H}-T_{C}$. Table 1 and Fig. 8 summarize the effect of $\Delta T$ on air motion near walls along longitudinal Lines $\mathrm{A}$ and B.

Figs. 7, e and $\mathrm{f}$ show profiles of temperature and horizontal velocity along Line $\mathrm{C}$. Temperature is increasing from the cold plate temperature on the bottom of the nacelle to reach a maximal value in the upper part of the nacelle ( $80 \%$ of the nacelle height), confirming again the adopted Boussinesq approximation, then it decreases to the cold plate temperature at the top wall of the nacelle. As it would be expected, horizontal velocity profiles along Line $\mathrm{C}$ show that motion of air is more significant in the vicinity of the top wall of the nacelle (cf. Fig. 7, f).

\subsection{Boundary layer analysis}

For further verification of the proposed numerical method and accuracy of the obtained results, it is of interest to put light on the boundary layer aspect. For this aim, we focus our study on air flow and heat transfer in the vicinity of the generator left wall. This choice is based on the relatively long distance between hot and cold plates on this side of the nacelle, which produces almost-quiescent air conditions far from the vertical wall. This physical situation is typically similar to the classical problem of Laminar Natural Convection flow and heat transfer about an Isothermal Vertical Plate (LNCIVP) treated by Ostrach using the semi-analytical method of similarity [8].

Along Line A and near the hot plate region, temperature and vertical velocity are, first, extracted and shifted to the $(\tilde{x}, \tilde{y})$ coordinate system (cf. Fig. 2), second, non-dimensionalized according to Eqs. (6) and (7), respectively, then plotted versus the non-dimensional modified ordinate $y^{*}$ given by Eq. (8).

$$
\begin{aligned}
& T^{*}=\left(T-T_{\infty}\right) /\left(T_{H}-T_{\infty}\right), \\
& v^{*}=v /\left(\frac{2 v G r_{\tilde{x}}^{0.5}}{\tilde{x}}\right), \\
& y^{*}=\tilde{y} /\left[\tilde{x}\left(\frac{4}{G r_{\tilde{x}}}\right)^{0.25}\right],
\end{aligned}
$$

where $T_{\infty}$ denotes the almost-quiescent air temperature far from the hot plate, that can be read directly from Fig. 7, a at $x=0.2 \mathrm{~m}$, and $G r_{\tilde{x}}$ is the Grashof number based on the modified abscissa $\tilde{x}$ given by:

$$
G r_{\tilde{x}}=\frac{g \beta\left(T_{H}-T_{\infty}\right) \tilde{x}^{3}}{v^{2}} .
$$




$-T_{\mathrm{C}}=-20^{\circ} \mathrm{C} \_T_{\mathrm{C}}=-10^{\circ} \mathrm{C} \_T_{\mathrm{C}}=00^{\circ} \mathrm{C}-T_{\mathrm{C}}=10^{\circ} \mathrm{C} \_T_{\mathrm{C}}=20^{\circ} \mathrm{C} \_T_{\mathrm{C}}=30^{\circ} \mathrm{C}$
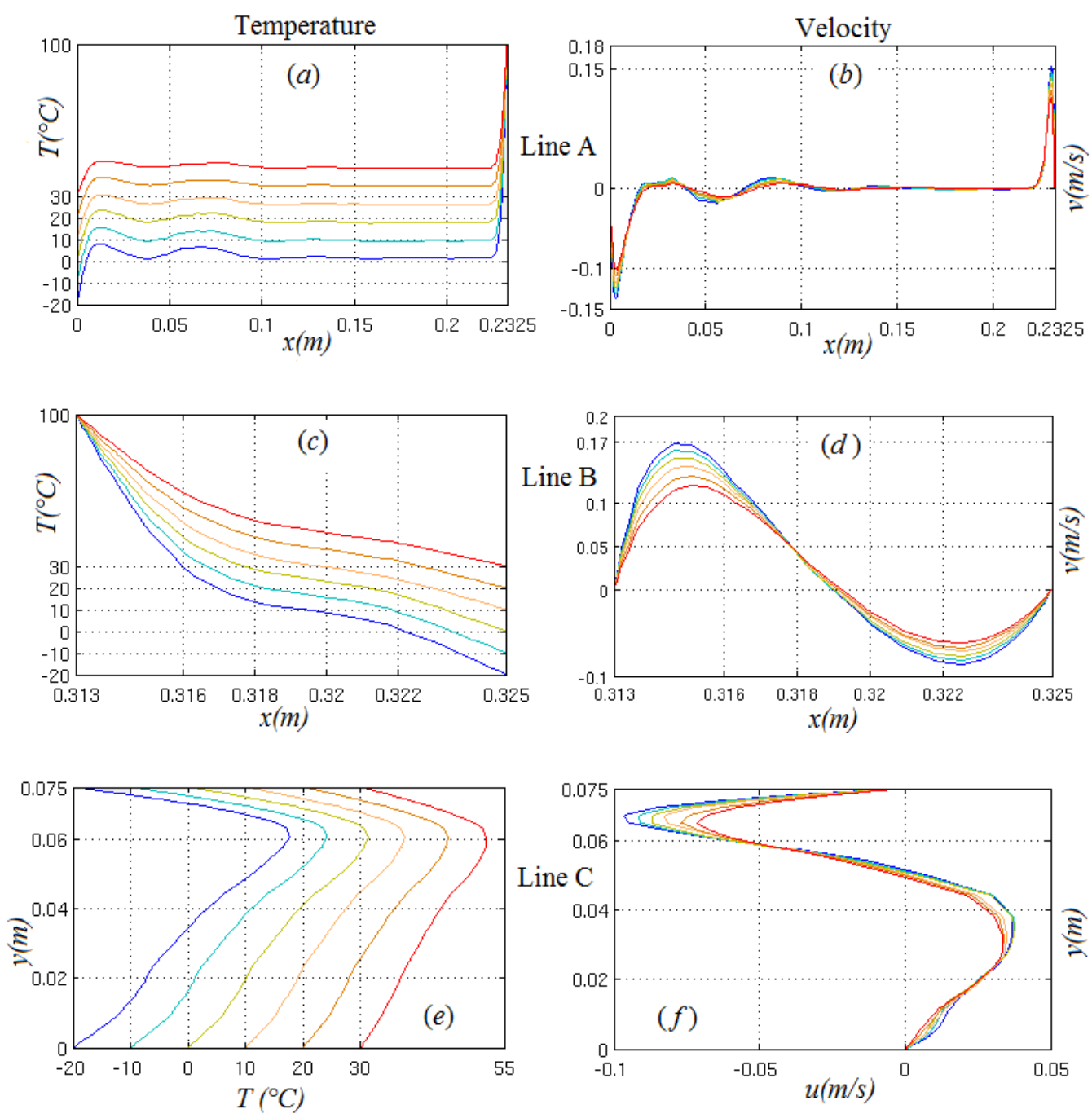

Fig. 7 Distributions of (a, c, e) temperature, (b, d) vertical and (f) horizontal velocity along Lines A, B and C, respectively

Figs. 9 and 10 illustrate, respectively, the obtained profiles of non-dimensional temperature and vertical velocity, for three different cooling temperature cases, along with the semi-analytical solution of LNCIVP problem, for the corresponding Prandtl number of the air.As it can be seen, the resulting non-dimensional temperature and vertical velocity profiles exhibit clearly a laminar boundary layer behavior, again confirming the proposed numerical approach.

Furthermore, for three $T_{C}$ values, the corresponding temperature and vertical velocity profiles are all collapsing on one curve, which validates the proposed non-dimensionalizing procedure. On the other hand, we notice a sharper over-estimating in the non-dimensional vertical velocity than it is the case in the non-dimensional temperature profile where a slight discard is obtained between our results and the semi-analytical solution. This discrepancy might be explained by the fact that air within nacelle far from the left side of the generator is not perfectly quiescent.

\subsection{Nacelle thermal behavior analysis}

To assess properly the hypothetical nacelle thermal behavior, both the required cooling capacity, $q_{C}$, and the resulting temperature-level inside the nacelle determined by the average air temperature, $T_{a v}$, constitute the key parameters. Since the heat exchange with the external environment has been neglected, the required cooling capacity reduces to the total heat rate inside the nacelle, i.e., $q_{\text {in }}$.

Thus, the energy balance is simplified into Equation (10), where $q_{\text {in }}$ is given by Eq. (5):

$$
q_{C}=q_{i n} .
$$

On the other hand, average temperature within the nacelle is calculated as:

$$
T_{a v}=\frac{1}{A} \int_{A} T(x, y) d A,
$$

where: $A$ denotes the inner surface area of the $2 \mathrm{D}$-nacelle. 
Table 1

Effect of temperature gap $\Delta T=T_{H}-T_{C}$ on air vertical velocity near walls along Lines $\mathrm{A}$ and $\mathrm{B}$

\begin{tabular}{|c|c|c|c|c|c|}
\cline { 2 - 6 } \multicolumn{2}{c|}{} & \multicolumn{2}{c|}{ Line A $(\mathrm{m} / \mathrm{s})$} & \multicolumn{2}{c|}{ Line B $(\mathrm{m} / \mathrm{s})$} \\
\hline$T_{C}\left({ }^{\circ} \mathrm{C}\right)$ & $\Delta T\left({ }^{\circ} \mathrm{C}\right)$ & $\left|v_{\max }^{H}\right|$ & $\left|v_{\max }^{C}\right|$ & $\left|v_{\max }^{H}\right|$ & $\left|v_{\max }^{C}\right|$ \\
\hline-20 & 120 & 0.1531 & 0.1371 & 0.1679 & 0.0866 \\
\hline-10 & 110 & 0.1462 & 0.1310 & 0.1600 & 0.0819 \\
\hline 0 & 100 & 0.1395 & 0.1241 & 0.1511 & 0.0770 \\
\hline 10 & 90 & 0.1316 & 0.1167 & 0.1414 & 0.0713 \\
\hline 20 & 80 & 0.1228 & 0.1086 & 0.1300 & 0.0677 \\
\hline 30 & 70 & 0.1137 & 0.1009 & 0.1195 & 0.0624 \\
\hline & $\left|\overline{v_{\max }}\right|$ & $\mathbf{0 . 1 3 4 5}$ & $\mathbf{0 . 1 1 9 7}$ & $\mathbf{0 . 1 4 5 0}$ & $\mathbf{0 . 0 7 4 5}$ \\
\cline { 2 - 6 } & $\Delta\left|v_{\max }\right|$ & 0.0394 & 0.0362 & $\mathbf{0 . 0 4 8 4}$ & $\mathbf{0 . 0 2 4 2}$ \\
\cline { 2 - 6 } & \multicolumn{2}{|c|}{} & & &
\end{tabular}

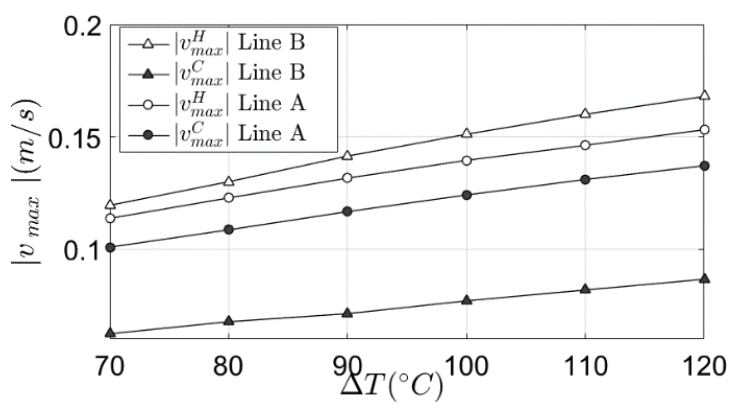

Fig. 8 Effect of DT on air vertical velocity near walls along Lines A and B

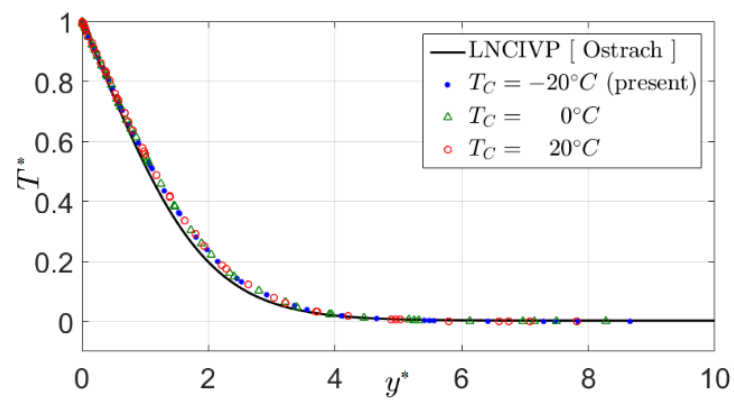

Fig. 9 Non-dimensional temperature profiles within the boundary layer

Figs. 11 and 12 show the resulting required cooling capacity and the average temperature within the nacelle as functions of the cooling temperature, $T_{C}$, along with the same parameters assessed by neglecting the buoyancy effect (i.e., pure conduction case). As it can be seen, in both cases, these two parameters exhibit a linear variation with respect to the cooling temperature.

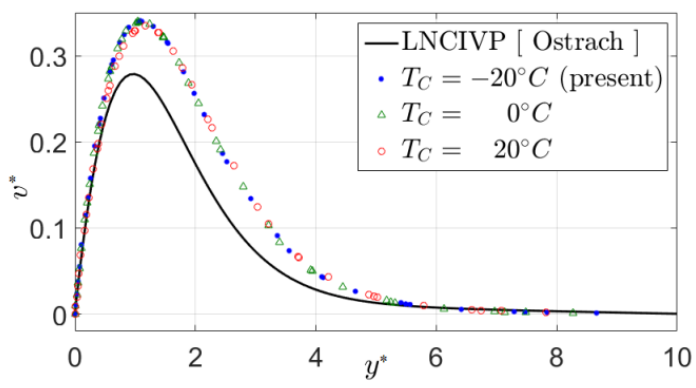

Fig. 10 Non-dimensional vertical velocity profiles within the boundary layer

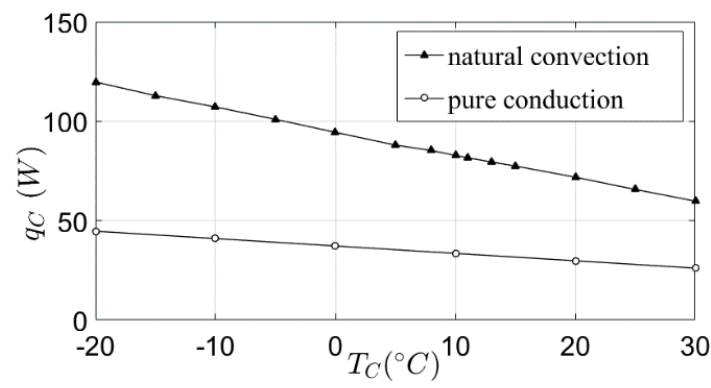

Fig. 11 Evolution of the cooling capacity with respect to the cooling temperature, with and without buoyancy effect

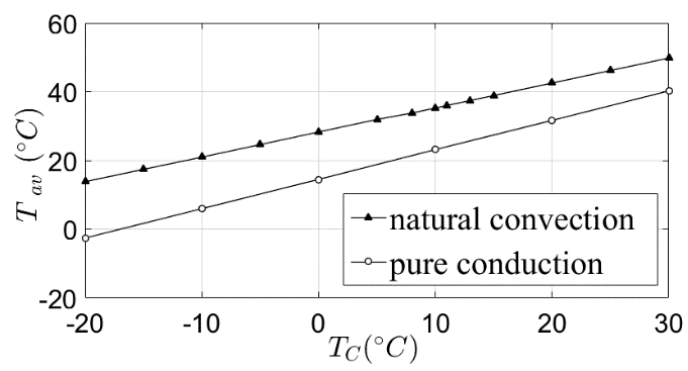

Fig. 12 Evolution of the average temperature with respect to the cooling temperature, with and without buoyancy effect

As it would be expected, the cooling process requires much more capacity rate in the natural convection case, where resulting temperature level is more significant. Furthermore, from Fig. 11, it is clear that the lower is the cooling temperature; the more energy-requiring is the cooling process. This is again a qualitative validation of the numerical method. We observe also that the buoyancy impact on the nacelle thermal behavior is intensifying as the temperature span, $\Delta T=T_{H}-T_{C}$, increases. Therefore, buoyancy effect might constitute a determinant factor for the thermal design of the nacelle cooling system.

\section{Conclusion}

This paper presents a numerical method to investigate the thermal behavior of a nacelle under the effect of natural convection heat exchange. For this purpose, a hypothetical nacelle has been considered, where external heat exchange and air flow around the nacelle have been neglected. The heat generation and the appropriate cooling system idealized as isothermal conditions have been represented respectively by hot and cold plates in the computational domain.

Simulations have been carried out for different cold plate temperatures. The resulting temperature and velocity profiles have been visualized along different directions within the nacelle, and have shown good qualitative agreement with the physical expectations.

As for validation, boundary layer analysis has been carried out by comparing the obtained numerical results with the semi-analytical solution of the classical problem of laminar natural convection about an isothermal vertical plate, where a satisfying agreement has been noticed. 
The impact of buoyancy forces on the thermal behavior of the hypothetical nacelle has been assessed by considering the required cooling capacity and the average temperature within the nacelle. Comparison with pure conduction case has been performed, and confirmed that appropriate values of required cooling loads are definitely dependent on the accurate predictions of natural convection within the nacelle.

Future works will be mainly focused on the development of more detailed numerical method that takes into account most of practical and nacelle thermal design considerations; such as the effects of heat exchange by radiation, modeling of heat generation (due to generator, gearbox and electronic components), three-dimensional flow fields within nacelle and around wind turbine, as well as turbulence modeling.

\section{Acknowledgment}

Computer Science and Engineering Department of University of Minnesota and the Algerian Government are gratefully acknowledged for their resources and sponsorship. Y. Saad, Distinguished Professor at UMN, USA, is particularly acknowledged for his valuable comments and support.

\section{References}

1. Smaili, A.; Masson, C.; Taleb, S.R.; Lamarche, L. 2006. Numerical study of the thermal behaviour of wind turbine Nacelle operating in Nordic climate, Numerical Heat Transfer Part B: Fundamentals 50(2): 121-141. http://dx.doi.org/10.1080/10407780600599547.

2. Tran, L. D.; Masson, C.; Smaili, A. 2006. A Stable Second-Order Mass-Weighted Upwind Scheme for Unstructured Meshes, International Journal for Numerical Methods in Fluids 51: 749-771. http://dx.doi.org/10.1002/fld.1146.

3. Smaili, A.; Tahi, A.; Masson, C. 2012. Thermal Analysis of Wind Turbine Nacelle Operating in Algerian Saharan Climate, Energy Procedia 18: 187-196. http://dx.doi.org/10.1016/j.egypro.2012.05.030.

4. Xydis, G.; Pechlivanoglou, G.; Nayeri, N.C. 2015. Wind Turbine Waste Heat Recovery-A Short-Term Heat Loss Forecasting Approach, Challenges 6: 188-201. http://dx.doi.org/10.3390/challe6020188.

5. Arzbaecher, C.; Parmenter, K.; Fouche, E. 2007. Industrial Waste-Heat Recovery: Benefits and Recent Advancements in Technology and Applications, 2007 ACEEE Summer Study on Energy Efficiency in Industry; European Council for an Energy Efficient Economy, Stockholm, Sweden.

6. Tammelin, B.; Cavaliere, M.; Holttinen, H.; Morgan, C.; Seifert, H.; Säntti, K. 2000. Wind Energy Production in Cold Climate: Meteorological Publications, Finnish Meteorological Institute, 41, Helsinki.
7. Catton, I. 1978. Natural Convection in Enclosures: Proc. 6th Int. Heat Transfer Conf., Toronto, Canada, 6:13-31.

8. Ostrach, S. 1953. An Analysis of Laminar Free Convection Flow and Heat Transfer about a Flat Plate Parallel to the Direction of the Generating Body Force: National Advisory Committee for Aeronautics, Report 1111: 63-79.

9. Bergman, T.L.; Lavine, A.S.; Incropera, F.P.; Dewitt, D.P. 2011. Fundamentals of Heat and Mass Transfer: Seventh edition, John Wiley \& Sons.

\author{
M.A. Mahdi, A. Smaili
}

\section{NUMERICAL INVESTIGATIONS OF LAMINAR BUOYANT HEAT TRANSFER IN A 2D-ENCLOSURE - APPLICATION TO WIND TURBINE NACELLE OPERATING IN HOT CLIMATE}

S u m m a r y

The climate of the Algerian Sahara is characterized by extreme thermal conditions. The wind turbines installed in the Sahara, namely the electromechanical equipment located inside the nacelle (e.g. electrical generator), are subjected to high and fluctuating temperature gradients during the day and the seasons. This reality might generate contradictory constraints of design and can lead to some difficulties of conception. In order to maintain an appropriate temperature of the air inside the nacelle (i.e. acceptable average temperature of the electromechanical equipment), the heat generated by the generator must be rejected towards the environment, and the resulting heat transfer must be controlled properly. This work deals with heat transfer effects within a reduced scale nacelle, i.e. hypothetical nacelle. For this purpose, a small nacelle dimensions have been considered and represented as rectangular cavity. In this paper, the air flow fields within the nacelle have been described using the Navier-Stokes equations. The energy equation has been used to account for heat transfer effects. ANSYS FLUENT code has been employed to solve the resulting governing equations. The main object of this work is to determine the average temperature inside the nacelle, as well as to investigate its variation with the required cooling capacity.

Keywords: Saharan climate, wind turbine nacelle, natural convection, cooling system.

Received July 30, 2016

Accepted October 13, 2017 